\title{
DOA Estimation with Non-Uniform Linear Arrays: A Phase-Difference Projection Approach
}

\author{
Hui Chen, Tarig Ballal, and Tareq Y. Al-Naffouri
}

\begin{abstract}
Phase wrapping is a major problem in directionof-arrival (DOA) estimation using phase-difference observations. For a sensor pair with an inter-sensor spacing greater than half of the wavelength $(\lambda / 2)$ of the signal, phase wrapping occurs at certain DOA angles leading to phase-difference ambiguities. Existing phase unwrapping methods exploit either frequency or spatial diversity. These techniques work by imposing restrictions on the utilized frequencies or the receiver array geometry. In addition to sensitivity to noise and calibration errors, these methods may also have high computational complexity. We propose a grid-less phase-difference projection (PDP) DOA algorithm to overcome these issues. The concept of wrapped phased-difference pattern (WPDP) is introduced, which allows the proposed algorithm to compute most of the parameters required for DOA estimation in an offline manner, hence resulting in a superior computational speed in realtime. Simulation results demonstrate the excellent performance of the proposed algorithm, both in terms of accuracy and speed.
\end{abstract}

Index Terms-Direction of arrival, phase-difference, phase ambiguity, non-uniform linear arrays, CRLB.

\section{INTRODUCTION}

Direction-of-arrival (DOA) estimation is an important topic for applications such as wireless sensor networks [1], indoor positioning and tracking [2], Radar [3], wireless communications [4], and so on. Many DOA estimation methods have been proposed over the years, as reviewed in [5], [6]. The literature focuses largely on the narrow-band (or single-frequency) case with uniform linear array (ULA) configurations. Extension to multi-frequency scenarios is usually straightforward. On the other hand, the use of non-uniform linear arrays (NULAs) is also popular. NULA configurations are often utilized to extend the array aperture and consequently improve the array's DOA resolution.

In this letter, we focus on single-source DOA estimation with NULAs. This scenario is motivated by a mmWave/THz multiple-input multiple-output (MIMO) communication context. In these systems, flexible arrays are adopted to alleviate the high computation and hardware costs (see switch-based MIMO [7] and array-of-subarray (AOSA) structures [8]). An antenna/subarray selection algorithm is applied, which often results in a NULA structure. These systems also employ beamforming to reduce interference and provide a dominant line-of-sight (LOS) signal [9]. Hence, DOA estimation for a single source observed at a NULA is an important problem for mmWave/THz MIMO systems.

The authors are with the Division of Computer, Electrical and Mathematical Science \& Engineering, King Abdullah University of Science and Technology (KAUST), Thuwal, 23955-6900, KSA. e-mail: (\{hui.chen; tarig.ahmed; tareq.alnaffouri\}@kaust.edu.sa).
The DOA estimation problem can be formulated as an optimization of a cost function over a feasible DOA range. Usually, the process requires evaluating the cost function for the whole DOA range, searching for that function's optimum. Maximum likelihood estimation (MLE) [10] and MUSIC [11] are two widely used methods that exemplify this approach. A drawback of this approach is that the search process can increase the computational complexity, especially when high spatial resolution is desired.

Time-delay estimation is a fast alternative solution to DOA estimation that can directly (without search) produce a DOA estimate [12]. The linear relationship between time delay and phase-difference makes it possible to utilize phase-difference measurements for DOA estimation. Phase-difference based DOA estimation has been reported as an effective approach for multi-carrier signals [13]. Nevertheless, phase-difference based DOA estimation suffers from the occurrence of phase wrapping [14]-[16].

The issue of phase wrapping can be resolved by exploiting the frequency diversity available in multi-frequency signals [17], [18], while in single-frequency scenarios, the focus of this paper, spatial diversity can be leveraged. Examples of spatial-diversity phase unwrapping methods include [14], [19], and the more recent $2 q$-order difference-set approach [20]. A major drawback of these methods is that they require a specialized sensor setup. Besides, these methods tend to be sensitive to the phase noise effect.

This letter proposes a phase-difference projection (PDP) method for DOA estimation using non-uniform linear arrays. The proposed method can be applied to an arbitrary linear array configuration of more than two sensors. Simulation results demonstrate that the proposed method outperforms a host of benchmark algorithms in terms of the root mean squared error (RMSE).

The structure of this work is organized as follows. Section II presents the observation model, and Section III presents the proposed PDP method for DOA estimation. Simulations are presented in Section IV before drawing conclusions in Section $\mathrm{V}$.

\section{ObSeRVATION Model}

We consider a complex sinusoidal source signal, with a frequency $f$ and amplitude $A, s(t)=A e^{-j 2 \pi f t}$ in the far field [21] of a non-uniform linear array of $N$ sensors. The source impinges on the array from a direction $\theta \in$ $(-\pi / 2, \pi / 2)$ rad. By using $d_{u v}$ to denote the distance between two sensors $(u$ and $v$ ) normalized by $\lambda / 2$, where $\lambda$ is the 
signal wavelength, the received signal (vector) at time $t$ can be modelled as [22]

$$
\mathbf{x}(t)=\mathbf{a}(\theta) s(t)+\mathbf{w}(t),
$$

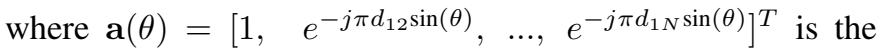
array steering vector, and $\mathbf{w}(t)$ is a vector of the additive noise.

The principal phase-difference across a sensor pair, $u$ and $v$, can be estimated from the $u$-th and $v$-th elements of $\mathbf{x}$ as

$$
\hat{\psi}_{u v}(t)=\operatorname{angle}\left(x_{u}(t) \cdot x_{v}^{*}(t)\right) \in[-\pi, \pi),
$$

where $(\cdot)^{*}$ is the complex conjugate operation. For simplicity, and without loss of generality, we will focus on singlesnapshot scenarios. Hence, we will drop the time variable $t$.

To develop our proposed method, we start from noise-free principal phase observations, $\psi_{u v}(\theta)$. These are related to the actual phase-difference, $\phi_{u v}(\theta)=\pi d_{u v} \sin (\theta)$ through

$$
\psi_{u v}(\theta)=\bmod \left(\phi_{u v}(\theta)+\pi, 2 \pi\right)-\pi=\pi d_{u v} \sin (\theta)-2 \pi q_{u v},
$$

where $\bmod (\cdot, \cdot)$ is the modulus operation, and $q_{u v}$ is an integer value given by the rounding operation

$$
q_{u v}=\operatorname{round}\left(\frac{\pi d_{u v} \sin (\theta)}{2 \pi}\right) .
$$

Based on (3), we observe that estimating the DOA from $\psi_{u v}(\theta)$ requires knowledge of the integer $q_{u v}$, which may not be available if a method such as (2) is used to estimate $\psi_{u v}(\theta)$. When $d_{u v} \leq 1, q_{u v}=0$ for any $\theta$. However, for $d_{u v}>1$, the latter result is not guaranteed, except for a specific range of $\theta$ values. Since $\theta$ is unknown, $\psi_{u v}(\theta)$ will always be ambiguous for $d_{u v}>1$.

\section{The Proposed PDP Algorithm}

\section{A. Wrapped Phase-Difference Pattern (WPDP)}

For an arbitrary source location $\theta \in[-\pi / 2, \pi / 2]$, using (3), we can compute the principal phase-difference across receiver pairs to create a (principal) phase-difference (PD) vector $\boldsymbol{\psi}(\theta)=\left[\psi_{u v}(\theta)\right]^{T}, u, v \in\{1, \cdots N\}, u<v$. Assuming that we utilize $M \leq\left(\begin{array}{c}N \\ 2\end{array}\right)$ sensor pairs, we can simplify the notations and write $\boldsymbol{\psi}(\theta)=\left[\psi_{m}(\theta)\right]^{T}$, and $\mathbf{q}(\theta)=$ $\left[q_{m}(\theta)\right]^{T}, m=1, \cdots, M$. We can also arrange the inter-sensor distances that corresponds to $\boldsymbol{\psi}(\theta)$ in a vector $\mathbf{d}=\left[d_{m}\right]^{T}$.

Now, let us think of $\psi(\theta)$ as a point in an $M$-dimensional space. From (3), and for $\theta=0$, we can see that $\psi_{m}(\theta)=$ $0, q_{m}(\theta)=0, \forall m \in\{1, \cdots, M\}$. By gradually increasing $\theta$ starting from $\theta=0$, we can see that $\psi_{m}(\theta)$ increase in proportion to one another. The value of the vector $\mathbf{q}(\theta)$ remains constant up to a certain $\theta$ value. Depending on the corresponding inter-sensor spacing $d_{m}$, an entry of $\mathbf{q}(\theta)$ may repeatedly increase by a value equal to one as $\theta$ increases. The opposite happens when $\theta$ changes in the negative direction. This creates different intervals of $\theta$, each interval with a distinct vector $\mathbf{q}$ that remains unchanged throughout the interval. Let us denote these intervals as $\Theta_{k}, k \in\{1, \cdots, K\}$. For any $\theta_{a}, \theta_{b} \in \Theta_{k}$,

$$
\mathbf{q}\left(\theta_{a}\right)=\mathbf{q}\left(\theta_{b}\right)=\mathbf{q}_{k},
$$

and based on (3), we can write

$$
\delta_{a b}=\boldsymbol{\psi}\left(\theta_{a}\right)-\boldsymbol{\psi}\left(\theta_{b}\right)=\pi \mathbf{d}\left[\sin \left(\theta_{a}\right)-\sin \left(\theta_{b}\right)\right] .
$$

The values of $\mathbf{q}(\theta)$ is the same (equals to $\mathbf{q}_{k}$ ) for all $\theta \in \Theta_{k}$, and so (6) is valid for any choice of $\left\{\theta_{a}, \theta_{b}\right\} \in \Theta_{k}$. This indicates that each continuum given by $\boldsymbol{\psi}\left(\Theta_{k}\right), k \in\{1 \cdots, K\}$, is a straight line; and that all the $K$ straight lines point in the same direction $\pm \mathbf{d} /\|\mathbf{d}\|_{2}$, where $\|\cdot\|_{2}$ is the Euclidean norm. That is we have $K$ parallel lines in $M$-dimensional space. The number of lines $K$ can be calculated as [13]

$$
K=2 \sum_{i=1}^{M} \operatorname{ceil}\left(\frac{\psi_{i}(\pi / 2)-\pi}{2 \pi}\right)+1,
$$

where ceil $(\cdot)$ returns the nearest integer greater than or equal to the argument. Based on (5), all points on the $k$-th WPD line can be compensated/unwrapped with the same unwrapping vector $\mathbf{h}_{k}=2 \pi \mathbf{q}_{k}$. That is

$$
\phi(\theta)=\boldsymbol{\psi}(\theta)+\mathbf{h}_{k}, \forall \theta \in \Theta_{k} .
$$

A procedure to compute $\mathbf{h}_{k}$ will be detailed in the next subsection. Fig. 1 shows an example plot of $\boldsymbol{\psi}\left(\Theta_{k}\right)$ for $M=2$. We refer to such a display as a wrapped phasedifference pattern (WPDP).

Using simple geometry, we can see that all the lines $\psi\left(\Theta_{k}\right)$ are perpendicular to a hyperplane that contains the origin. This hyperplane is defined by the equation

$$
\mathbf{d}^{T} \boldsymbol{\psi}(\theta)=d_{1} \psi_{1}(\theta)+d_{2} \psi_{2}(\theta)+\cdots+d_{M} \psi_{M}(\theta)=0 .
$$

Each line $\psi\left(\Theta_{k}\right)$ coincides with a projection point, $\mathbf{p}_{k}$, where the line intersects with the hyperplane. In the following discussion, we show how to obtain these projection points.

Given a principal observation vector $\psi(\theta)$, the distance between this vector, treated as a point in $M$-dimensional space, and the hyperplane (9), is given by

$$
\operatorname{dist}\left(\boldsymbol{\psi}(\theta), \mathbf{d}^{T} \boldsymbol{\psi}=0\right)=\frac{\mathbf{d}^{T} \boldsymbol{\psi}(\theta)}{\|\mathbf{d}\|_{2}}
$$

The projection point $\mathbf{p}(\theta)$ of $\boldsymbol{\psi}(\theta)$ on the hyperplane along the direction $\mathbf{d} /\|\mathbf{d}\|$ can be obtained as

$$
\mathbf{p}(\theta)=\operatorname{project}(\boldsymbol{\psi}(\theta))=\boldsymbol{\psi}(\theta)-\frac{\mathbf{d}^{T} \boldsymbol{\psi}(\theta)}{\|\mathbf{d}\|_{2}} \cdot \frac{\mathbf{d}}{\|\mathbf{d}\|_{2}} .
$$

It is obvious that all points on the same WPD line are projected to the same point on the projection hyperplane. However, for an observed noisy wrapped phase-difference vector $\hat{\psi}, 11$ returns only a perturbed projection point $\hat{\mathbf{p}} \notin\left\{\mathbf{p}_{k}\right\}$. In this case, we pick the nearest projection point $\mathbf{p}_{z}$, where

$$
z=\underset{k}{\arg \min }\left\|\mathbf{p}_{k}-\hat{\mathbf{p}}\right\|_{2} \text {. }
$$

Now, the unbiased WPD $\tilde{\psi}$, which is the nearest point on the line with the projection point $\mathbf{p}_{k}$ can be estimated as

$$
\tilde{\boldsymbol{\psi}}=\mathbf{p}_{z}+\frac{\mathbf{d}^{T} \hat{\boldsymbol{\psi}}}{\|\mathbf{d}\|_{2}} \cdot \frac{\mathbf{d}}{\|\mathbf{d}\|_{2}} .
$$

An illustration of a noise-free WPDP for an array of 3 elements is depicted in Fig. 1 . The sensors are separated by spacings of $\mathbf{r}=[0, \Delta, \Delta(1+\delta)]=[0,2.3,5.18](\lambda / 2$ units, 
measured from the first sensor, where $\Delta=2.3, \delta=1.25$ ). Each WPDP point is formed as $\boldsymbol{\psi}(\theta)=\left[\psi_{12}(\theta), \psi_{23}(\theta)\right]^{T}$. In this figure, $K=5$ WPD lines are displayed together with the corresponding projection points, $\mathbf{p}_{k}(k=1, \cdots, 5)$. In this 2-dimensional WPDP, the projection hyperplane is actually a line. The $\theta$ values for selected points are also quoted. We note that for the middle line $\left(\theta \in\left[-20^{\circ}, 20^{\circ}\right]\right), \psi(\theta)=\phi(\theta)$, i.e., no phase-difference wrapping is occurring. For this line, $\psi_{2}$ reaches the value $\pi$ at around $\theta=20^{\circ}$, resulting in a jump or wrapping when $\theta$ increases slightly. For the rest of the points that are located on the other lines, $\psi(\theta) \neq \phi(\theta)$ due to phase wrapping. Some compensation is needed to be able to perform DOA estimation. The following subsection addresses this issue.

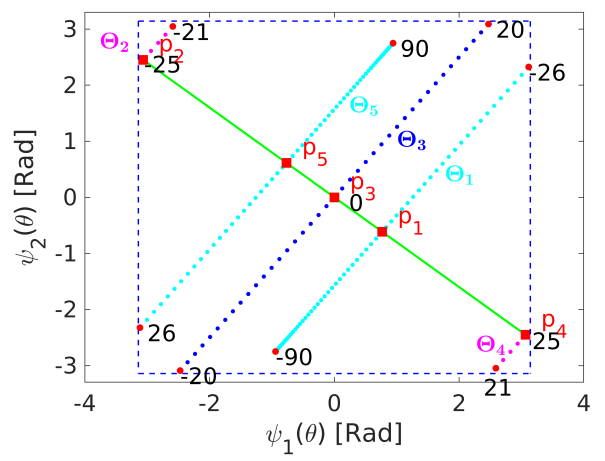

Fig. 1. An example of a WPDP for $M=2, \Delta=2.3, \delta=1.25$.

\section{B. Unwrapping Vectors}

The unwrapping vector $\mathbf{h}_{k}$ can be obtained by tracing the WPD lines. Together with their projection points, these lines are easily identified by their (known) direction unit vectors and starting points. We can start from the point $\psi(-\pi / 2)$, which, let us say, falls on the first line. The point where this line intersects with the M-cube, whose boundaries are $-\pi$ and $\pi$, can easily be calculated. The intersection point determines the next line's starting point, which is obtained by wrapping the coordinate of $\psi$ that crosses the cube's surface. A pseudocode for calculating $\mathbf{p}_{k}$ and $\mathbf{h}_{k}$ is listed in Algorithm 1

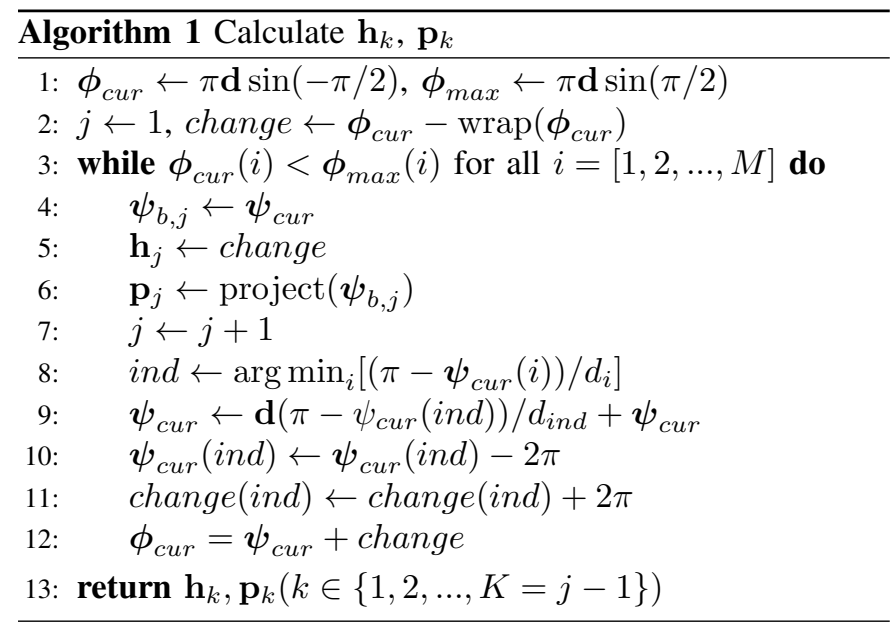

\section{The Proposed PDP DOA Estimation Algoirthm}

The proposed DOA algorithm starts by initializing $\mathbf{h}_{k}$ and $\mathbf{p}_{k}$ using algorithm 1 . Then, for a noisy WPD $\hat{\psi}$, the unbiased WPD $\tilde{\psi}$ can be obtained using (11)-(13). Next, the estimated unwrapped phase-difference vector $\phi$ can be obtained using (8). Finally, the DOA of the source can be calculated using (3). A pseudocode of the proposed DOA algorithm is listed in Algorithm 2. (Matlab codes available in Github).

Remark 1: In Algorithm 2, we note that the bulk of the computational complexity lies in Step-1. This step needs to be performed only once at the initial setup (offline). The rest of the algorithm (online) steps are very simple. This makes the proposed algorithm very computationally efficient.

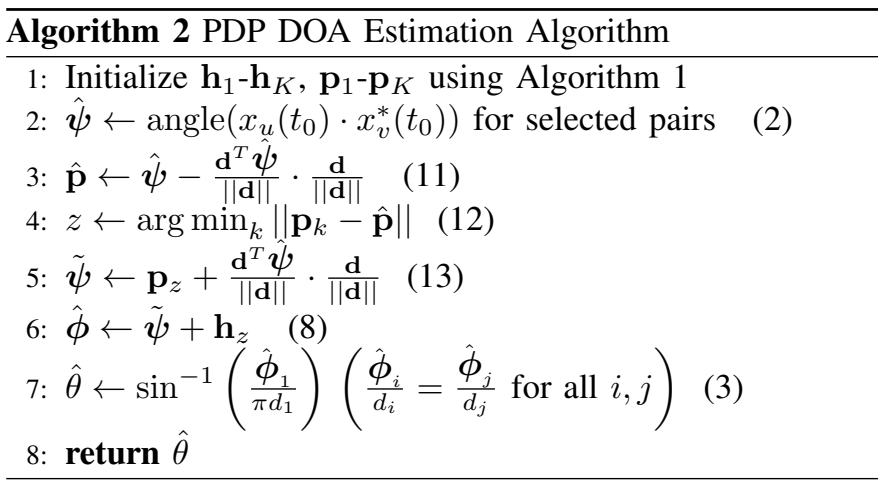

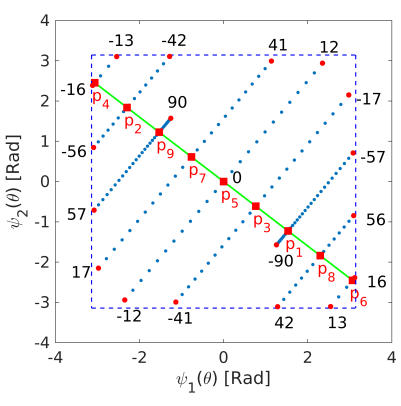

(a) $\Delta=3.6, \delta=1.25$

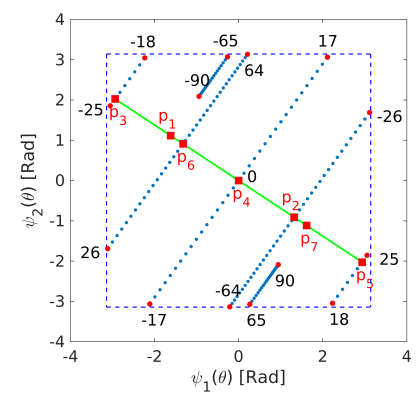

(b) $\Delta=2.3, \delta=1.45$
Fig. 2. Visualization of two different WPDPs.

Remark 2: Noise perturbation in WPD tends to drift a noisy WPD point away from its original (noise-free) WPD line. The proposed PDP algorithm associates a noisy point with the closest projection point. Depending on the noise level, this might lead to a wrong (hard) decision. For a specific signal frequency, the distance between the projection points is solely determined by the array layout, which can easily be deduced from (11). The characteristics of different array configurations based on their WPDP structures can provide an interesting tool for analyzing these arrays' behavior for different source locations. We illustrate the impact of array configuration in Fig. 2 which shows WPDP examples for two different array configurations with the same number of elements $(\mathbf{r}=[0, \Delta, \Delta(1+\delta)])$. From the figure, we can see a significant difference in the WPDP structures for the two arrays as reflected in the number of projection points 


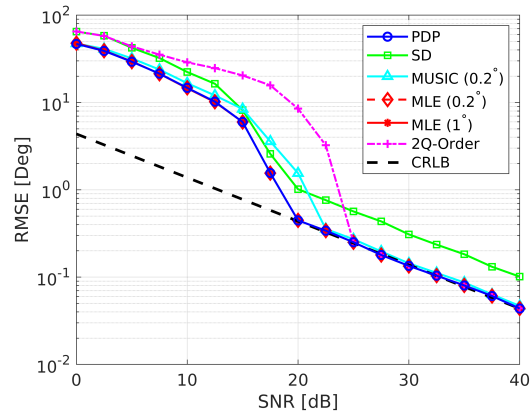

(a) $\mathbf{r}_{3}$

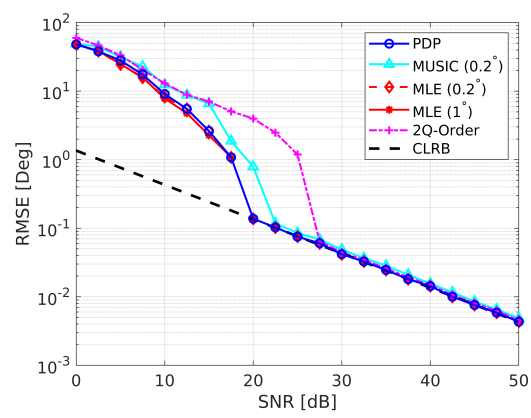

(b) $\mathbf{r}_{4}$

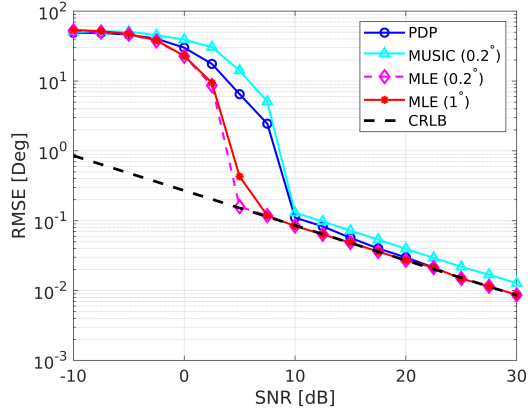

(c) $\mathbf{r}_{10}$

Fig. 3. RMSE for different DOA estimation algorithms tested over three different array configuration.

and the inter-point distances, which impacts DOA estimation performance. As an example, it is expected that recovering a projection point correctly from noisy observations to be easier for $\mathbf{p}_{4}$ in Fig. 2 (b) compared to the rest of the projection points in both Fig. 2 (a) and (b). On the other hand, in situations where two WPD lines overlap, the proposed algorithm, or any other DOA estimation algorithm, will fail to identify the source location. This happens when the array configuration is ambiguous for certain source locations [23]. PDP inherently requires the setup to be unambiguous.

\section{Simulation Results}

In this section, we evaluate the performance of the proposed PDP DOA estimation algorithm given in Algorithm 2. Simulations were carried out for this purpose. The proposed algorithm is compared with several benchmark methods, including the MUSIC algorithm [11], the spatial-diversity (SD) based algorithm [19], the 2q-order algorithm [20], maximum likelihood estimation (MLE) [10], in addition to the CramérRao lower bound (CRLB). For a given sensor layout, and a source location $\theta$, the CRLB is calculated as [10]

$$
\text { CRLB }=\frac{1}{2 \pi^{2} N S U \sin (\theta)}, \quad U=\frac{1}{N} \sum_{n=1}^{N}\left(r_{n}-\overline{\mathbf{r}}\right),
$$

where $S$ is the linear SNR, and $\mathbf{r}$ is an $N \times 1$ sensor spacing vector, $r_{n}$ and $\overline{\mathbf{r}}$ are the $n$-th element and the average value of $\mathbf{r}$, respectively.

To demonstrate the performance of various methods three different sensor layouts with different number of sensors $(N=3,4,10)$ are considered. Namely, we test $\mathbf{r}_{3}=$ $[0,2.4,5.4], \mathbf{r}_{4}=[0,5,10.5,16.5]$ (setup from [20]) and $\mathbf{r}_{10}=[0,1,6,10,23,26,34,41,53,55]$ (a non-redundant array from [24]), are shown in Fig. 3 The root mean squared error (RMSE) versus SNR is adopted as the performance metric, which is calculated from 5000 simulation trials at each SNR value. In each trial, the source location is generated randomly from a uniform distribution between $39.5^{\circ}$ to $40.5^{\circ}$. For the proposed method, $M=\left(\begin{array}{c}N \\ 2\end{array}\right)$ phase-difference estimates are computed using (2). For the MUSIC algorithm and MLE, to reduce the computational complexity, the search process is implemented in two stages [10]: a coarse search (using a $0.2^{\circ}$ or $1^{\circ}$ step) followed by a fine search (using a $0.02^{\circ}$ step). The
SD based algorithm works only for three sensors $\left(\mathbf{r}_{3}\right)$, while the $2 q$-order algorithm works for $\mathbf{r}_{3}$ and $\mathbf{r}_{4}$. In all simulation trials, and for all methods, a single snapshot is used to estimate the source location. Fig. 3 plots the RMSE performance, while Table I lists the average runtime, in milliseconds (ms).

For the configurations $\mathbf{r}_{3}$ and $\mathbf{r}_{4}$, the proposed PDP algorithm matches the RMSE of the MLE for the finer search grid, which coincides with the CRLB. PDP and MLE outperform all the other methods. The proposed algorithm also shows superiority in terms of computational complexity; it is substantially faster than both MLE and MUSIC. The SD and $2 q$-order algorithms are slightly faster than the proposed algorithm; however, their RMSE performance is inferior.

For the configuration $\mathbf{r}_{10}$, the proposed algorithm does not match the MLE performance but still outperforms MUSIC in terms of the RMSE. This time, when the coarse search is performed using a grid of $1^{\circ}$, MLE exhibits a slightly faster execution time compared to the proposed method. This can be attributed to the fact that, in this case, PDP uses all the pairwise combinations of sensors. Apparently, this is redundant since the number of pairs becomes excessively large as $N$ increases. The proposed method's computational complexity can be further reduced by judiciously choosing a sufficient subset of the sensor pairs to achieve adequate performance.

TABLE I

AVERAGE PROCESSING TIME OF DIFFERENT ALGORITHMS [MS]

\begin{tabular}{c|ccc}
\hline Algorithms & $\mathbf{M = 3}$ & $\mathbf{M = 4}$ & $\mathbf{M = 1 0}$ \\
\hline PDP & 0.024 & 0.041 & 1.084 \\
\hline SD & 0.008 & - & - \\
\hline MUSIC $\left(0.2^{\circ}\right)$ & 2.972 & 3.541 & 4.315 \\
\hline MLE $\left(0.2^{\circ}\right)$ & 2.003 & 2.123 & 2.515 \\
\hline MLE $\left(1^{\circ}\right)$ & 0.753 & 0.780 & 0.923 \\
\hline 2-Q Order & 0.004 & 0.020 & - \\
\hline
\end{tabular}

\section{CONCLUSION}

A phase-difference projection (PDP) based direction of arrival (DOA) algorithm is proposed. The proposed algorithm projects the phase-difference observations measured across sensor pairs on a pre-defined hyperplane determined by the array geometry. Based on this projection, DOA estimation can be achieved in a simple and computationally efficient manner. 
Simulation results show that the proposed algorithm can match maximum likelihood estimation and outperform MUSIC while maintaining a substantially higher computational speed.

The results can be further improved by utilizing multiple frequencies and multiple snapshots of the signal. Future directions include performance analysis and sensor-pair selection for larger receiver arrays.

\section{REFERENCES}

[1] S. Tomic, M. Beko, and R. Dinis, "3-D target localization in wireless sensor networks using RSS and AoA measurements," IEEE Transactions on Vehicular Technology, vol. 66, no. 4, pp. 3197-3210, 2016.

[2] L. Wan, G. Han, L. Shu, S. Chan, and T. Zhu, "The application of DOA estimation approach in patient tracking systems with high patient density," IEEE Transactions on Industrial Informatics, vol. 12, no. 6, pp. 2353-2364, 2016.

[3] J. Xu, W.-Q. Wang, and R. Gui, "Computational efficient DOA, DOD, and Doppler estimation algorithm for MIMO radar," IEEE Signal Processing Letters, vol. 26, no. 1, pp. 44-48, 2018.

[4] H. Huang, J. Yang, H. Huang, Y. Song, and G. Gui, "Deep learning for super-resolution channel estimation and DOA estimation based massive MIMO system," IEEE Transactions on Vehicular Technology, vol. 67, no. 9, pp. 8549-8560, 2018.

[5] H. Krim and M. Viberg, "Two decades of array signal processing research," IEEE signal processing magazine, 1996.

[6] T. E. Tuncer and B. Friedlander, Classical and modern direction-ofarrival estimation. Academic Press, 2009.

[7] S. Payami, N. M. Balasubramanya, C. Masouros, and M. Sellathurai, "Phase shifters versus switches: An energy efficiency perspective on hybrid beamforming," IEEE Wireless Communications Letters, vol. 8, no. 1, pp. 13-16, 2018.

[8] W. Huang, Z. Lu, Y. Huang, and L. Yang, "Hybrid precoding for single carrier wideband multi-subarray millimeter wave systems," IEEE Wireless Communications Letters, vol. 8, no. 2, pp. 484-487, 2018.

[9] H. Sarieddeen, M.-S. Alouini, and T. Y. Al-Naffouri, "An overview of signal processing techniques for terahertz communications," arXiv preprint arXiv:2005.13176, 2020.

[10] F. Athley, "Threshold region performance of maximum likelihood direction of arrival estimators," IEEE Transactions on Signal Processing, vol. 53, no. 4, pp. 1359-1373, 2005.

[11] R. Schmidt, "Multiple emitter location and signal parameter estimation," IEEE transactions on antennas and propagation, vol. 34 , no. 3, pp. 276280, 1986.

[12] L. Liu and H. Liu, "Joint estimation of DOA and TDOA of multiple reflections in mobile communications," IEEE Access, vol. 4, pp. 3815$3823,2016$.

[13] H. Chen, T. Ballal, X. Liu, and T. Y. Al-Naffouri, "Realtime 2-D DOA estimation using phase-difference projection (PDP)," in 2019 27th European Signal Processing Conference (EUSIPCO). IEEE, 2019, pp. $1-5$.

[14] T. Ballal and C. J. Bleakley, "DOA estimation of multiple sparse sources using three widely-spaced sensors," in 2009 17th European Signal Processing Conference. IEEE, 2009, pp. 1978-1982.

[15] Molaei, Amir Masoud, Bijan Zakeri, and Seyed Mehdi Hosseini Andargoli, "High-performance localization of mixed fourth-order stationary sources based on a spatial/temporal full esprit-like method," Signal Processing, vol. 171, p. 107468, 2020.

[16] Molaei, Amir Masoud, and Masoud Hoseinzade, "High-performance 2D DOA estimation and 3D localization for mixed near/far-field sources using fourth-order spatiotemporal algorithm," Digital Signal Processing, vol. 100, p. 102696, 2020.

[17] H. Chen, T. Ballal, M. Saad, and T. Y. Al-Naffouri, "Angle-of-arrivalbased gesture recognition using ultrasonic multi-frequency signals," in 2017 25th European Signal Processing Conference (EUSIPCO). IEEE, 2017, pp. 16-20.

[18] T. Ballal and C. J. Bleakley, "DOA estimation for a multi-frequency signal using widely-spaced sensors," in 2010 18th European Signal Processing Conference. IEEE, 2010, pp. 691-695.

[19] — - "Phase-difference ambiguity resolution for a single-frequency signal," IEEE Signal Processing Letters, vol. 15, pp. 853-856, 2008.

[20] Y. Li, X. Zou, B. Luo, W. Pan, L. Yan, and P. Liu, "A 2q-order difference-set approach to eliminate phase ambiguity of a singlefrequency signal," IEEE Signal Processing Letters, vol. 26, no. 10, pp $1526-1530,2019$.
[21] J. R. Gonzalez and C. J. Bleakley, "High-precision robust broadband ultrasonic location and orientation estimation," IEEE Journal of selected topics in Signal Processing, vol. 3, no. 5, pp. 832-844, 2009.

[22] C. Zhou, Y. Gu, X. Fan, Z. Shi, G. Mao, and Y. D. Zhang, "Directionof-arrival estimation for coprime array via virtual array interpolation," IEEE Transactions on Signal Processing, vol. 66, no. 22, pp. 5956-5971, 2018.

[23] A. Manikas and C. Proukakis, "Modeling and estimation of ambiguities in linear arrays," IEEE Transactions on Signal Processing, vol. 46, no. 8, pp. 2166-2179, 1998.

[24] E. Vertatschitsch and S. Haykin, "Nonredundant arrays," Proceedings of the IEEE, vol. 74, no. 1, pp. 217-217, 1986. 